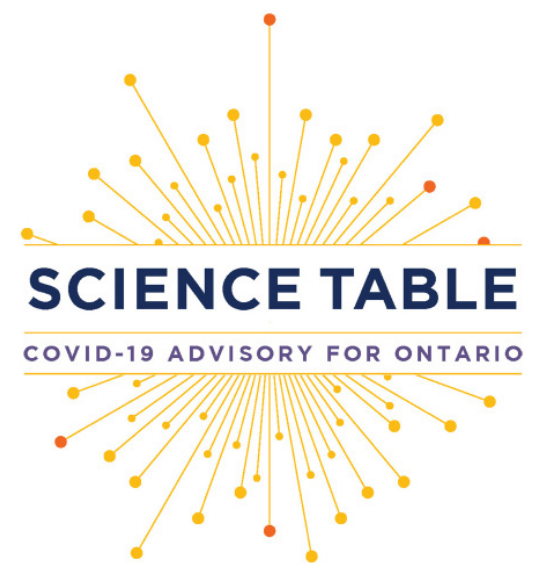

Version: 1.1

Published: June 23, 2021

Updated on June 23, 2021.

Citation: Presseau J, Arnason T, Buchan $\mathrm{JL}$ et al. Strategies to support Ontarians' capability, opportunity, and motivation for COVID-19 vaccination. Science Briefs of the Ontario COVID-19 Science Advisory Table. 2021;2(36). https://doi.org/10.47326/ ocsat.2021.02.36.1.0

Author Affiliations: The affiliations of the members of the Ontario COVID-19 Science Advisory Table can be found at https:// covid19-sciencetable.ca/.

Declarations of Interest: The declarations of interest of the members of the Ontario COVID-19 Science Advisory Table, its Working Groups, or its partners can be found at https:// covid19-sciencetable.ca/. The declarations of interest of external authors can be found under additional resources at https://doi. org/10.47326/ocsat.2021.02.36.1.0

About Us: The Ontario COVID-19 Science Advisory Table is a group of scientific experts and health system leaders who evaluate and report on emerging evidence relevant to the COVID-19 pandemic, to inform Ontario's response. Our mandate is to provide weekly summaries of relevant scientific evidence for the COVID-19 Health Coordination Table of the Province of Ontario, integrating information from existing scientific tables, Ontario's universities and agencies, and the best global evidence. The Science Table summarizes its findings for the Health Coordination Table and the public in Science Briefs.

The Behavioural Science Working Group is a group of scientific experts and public health leaders with specific expertise in behaviour change. Their expertise spans behavioural medicine, health, clinical and social psychology, behavioural economics, and implementation science. The Working Group evaluates emerging scientific evidence related to vaccination in healthcare workers and the general population, alongside other recommended protective behaviours such as physical distancing, mask-wearing, testing, and supported quarantine. The Working Group reports its findings to the public and the Science Table. Its findings are also

\section{Strategies to Support Ontarians' Capability, Opportunity, and Motivation for COVID-19 Vaccination}

Justin Presseau, Trevor Arnason, Judy L. Buchan, Rachel Burns, Kimberly M. Corace, Vinita Dubey, Gerald A. Evans, Leandre R. Fabrigar, Jeremy M. Grimshaw, Gabrielle M. Katz, Antonina Maltsev, Douglas G. Manuel, Rhiannon Mosher, Gilla Shapiro, Nathan M. Stall, Ashini Weerasinghe, Laura Desveaux on behalf of the Behavioural Science Working Group and the Ontario COVID-19 Science Advisory Table

\section{Key Message}

As of June 12, 2021, over 7 million (more than 51\%) Ontarians have been vaccinated with one dose of a COVID-19 vaccine and over 1 million (more than 12\%) have received two doses. ${ }^{1}$

Most (76\%) Ontarians report wanting to get a COVID-19 vaccine (Murty K, personal communication); however, getting vaccinated against COVID-19 requires more than motivation alone. A combination of capability, opportunity and motivation (key drivers of behaviour) are needed to ensure everyone who wants to get vaccinated, is able to do so. ${ }^{2}$ Of note, strategies to support these drivers of behaviour are not always available, or provided equally or consistently across Ontario. ${ }^{3,4}$

Behavioural science-informed strategies can address capability and opportunity barriers facing people who are already motivated to get vaccinated to get both doses of the COVID-19 vaccines. These strategies will be key to achieving the goal of a maximally vaccinated population. Among those with lower vaccine confidence, (Murty $\mathrm{K}$, personal communication) further addressing known motivation-related barriers can help support Ontarians in reaching the decision that is right for them. This includes leveraging trusted sources (such as health care professionals and community leaders) to address concerns about the speed of vaccine development and potential side effects, implementing supports to increase ease and opportunity for each dose, and employing effective communication (i.e., articulating when, where, and how) around available supports to enable capability and opportunity.

This brief is designed to complement and enhance existing vaccination rollout campaigns in Ontario. The intent is to provide behavioural science-informed insights into which strategies and policies can be leveraged to address multifaceted vaccination barriers, enabling Ontarians to get each dose. As vaccine supply continues to increase in the province, this strategy will accelerate Ontario's race to maximize immunity and support a post-pandemic Ontario. 
summarized in Science Briefs.

Correspondence to: Secretariat of the Ontario COVID-19 Science Advisory Table (info@covid19-sciencetable.ca)

Copyright: 2021 Ontario COVID-19 Science Advisory Table. This is an open access document distributed under the terms of the Creative Commons Attribution License, which permits unrestricted use, distribution, and reproduction in any medium, provided that the original work is properly cited.

The views and findings expressed in this Science Brief are those of the authors and do not necessarily reflect the views of all of the members of the Ontario COVID-19 Science Advisory Table, its Working Groups, and its partners.
5 Principles for Supporting the Decision to Get Vaccinated

5 Principles for Supporting Vaccination for Each Dose

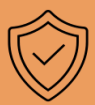

Capability

1 Provide reminders for each dose and create a plan to address anticipated obstacles to booking and attending.

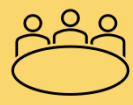

Opportunity

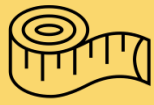

Build social norms through social comparison to similar others and leverage trusted sources (note these are different individuals in different communities).

Recognize the role that medical mistrust plays and allow time to address it.
2 Use opt-out strategies to nudge individuals to get vaccinated, rather than opt-in strategies.

3 Make booking and attendance easy

3 by maximizing use of accessible and convenient locations, times and days of the week. Implement flexible work hours and paid time off to encourage uptake.

One size will not fit all. Ensure 4 multiple strategies to reach those who are homebound or not comfortable with technology.

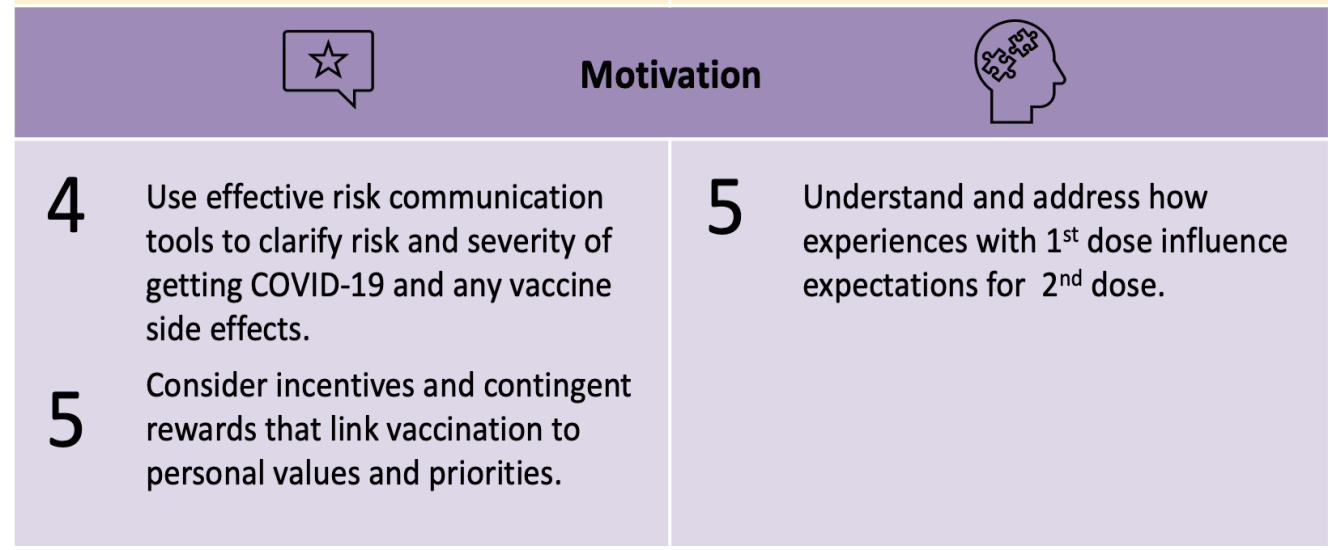

Figure 1. Ten Principles to Support Ontarians' Capability, Opportunity, and Motivation for COVID-19 Vaccination The clinical evidence and guidance on mixing vaccine brands and recommendations for particular brands continues to evolve based on international surveillance and research. However, the principles herein broadly hold and should be leveraged as evidence continues to develop.

\section{Background}

Ontario is in the midst of its COVID-19 vaccination roll-out while also experiencing the third and largest wave during the pandemic. Behavioural science provides insight into the WHAT and HOW of operationalizing Ontario's vaccine response. Specifically, behavioural science can support the implementation of necessary strategies at the individual level (i.e., addressing vaccination motivation) as well as structural strategies that enable vaccine uptake (i.e., the capability and opportunity barriers that prevent vaccination). 


\section{Questions}

What are known barriers and enablers to vaccination intention?

What are the known barriers and enablers to uptake of COVID-19 vaccines?

Which strategies are available to support vaccination intention and uptake of each dose of COVID-19 vaccines?

\section{Findings}

\section{Barriers and Enablers to Vaccination Intention and Uptake}

\section{Vaccine Intentions in Ontario}

Data from a survey of 18,482 Ontario residents aged 15 to 98 years collected from December 9, 2020 to March 15, 2021 (after Health Canada began approving COVID-19 mRNA vaccines) showed that $76 \%$ intended to get vaccinated against COVID-19. This rate has remained consistent over time (Murty $\mathrm{K}$, personal communication). While the majority of Ontarians intend to get vaccinated, reported intention varies across jurisdictions (from $72 \%$ in Toronto to $88 \%$ in Ottawa), age (82\% for those aged 55 years and older), and annual household income (from less than $70 \%$ in households with an income under $\$ 15,000$ CAD to $84 \%$ in households with an income from $\$ 90,000$ to $\$ 110,000$ CAD). While the majority of Ontarians (71\%) self-identifying as racialized reported intending to get vaccinated, this rate was lower than the overall vaccination intention (76\%) across the province. Less than $60 \%$ of respondents from households with at least four people unable to work remotely intend to get vaccinated, compared to $83 \%$ when all household members can work remotely.

\section{Barriers and Enablers to Vaccine Uptake}

A living behavioural science evidence synthesis of factors associated with vaccination intention and uptake by Crawshaw et al. (May 2021) identified 66 studies (1 in Canada) that collected data after COVID-19 vaccines were approved. ${ }^{5}$ The review categorized factors according to capability, opportunity and motivation factors, and highlighted capability (knowledge), opportunity (social influences and environmental context and resources) and motivation (beliefs about consequences) as primary barriers. These specific barriers directly inform the principles outlined below for addressing vaccine confidence and uptake. A key capability barrier included lack of knowledge about COVID-19 vaccines. Meanwhile, COVID-19 vaccine acceptance and uptake were enabled by disease-specific guidance on vaccination safety. Opportunity barriers to vaccine uptake were shown to be effectively mitigated by seeing others like oneself being vaccinated and through healthcare provider recommendations to get vaccinated. Lower vaccine confidence was associated with "allowing others to be vaccinated first" (this could be due to altruism or due to safety concerns). Mistrust in the government and health institutions was cited as a barrier to vaccine acceptance, especially among racialized groups. However, most survey data available to date do not provide specific detail on barriers or enablers for specific groups.

Access issues also posed barriers to vaccination in terms of the amount of time required to book and attend the vaccination appointment, convenience of clinic location, and direct costs associated with vaccination. ${ }^{5}$ Motivation-related barriers to vaccination uptake concerned perceptions of the safety and efficacy of vaccines, including the belief that the development of COVID-19 vaccines was rushed, mistrust of the medical community, and suspicion of political or economic influence. ${ }^{6}$ Some were worried about adverse reactions, specifically contraindications, and held the belief that natural immunity was better. ${ }^{5}$ 
What has Worked Locally and Internationally

Israel's vaccine rollout demonstrated the value of leveraging community-based resources and infrastructure to expedite vaccine distribution. ${ }^{7}$ One-third of nurses in Israel work in community-based settings, and engaging this population alongside primary care physicians, trained emergency medical technicians and paramedics helped accelerate the vaccine rollout. In Ontario, primary care physicians administer most annual influenza vaccinations and could be engaged, along with nurse practitioners and other community-based healthcare workers, to connect with hardto-reach communities and individuals. They are also a central resource in building vaccine confidence. The US expedited its own vaccine rollout by leveraging pharmacies and churches to enable easy administration. ${ }^{8}$ The Region of Peel applied similar principles in partnering with trusted community partners in places of worship (i.e., temples, mosques, gurdwaras) to establish and operate pop-up clinics to vaccinate its vulnerable populations - many of whom are immigrants and do not speak English. ${ }^{9}$ Peel also launched a 32-hour overnight vaccine clinic called "Doses After Dark" to increase accessibility to shift and other essential workers, with the aim of delivering more than 7,600 doses, including 4,939 doses administered during the overnight hours from 12:30 p.m. on Saturday, May 15 to 8:30 p.m. on Sunday, May $16 .{ }^{10}$

\section{Strategies to Support Vaccination Intention and Uptake}

\section{Vaccination Intention} Barriers/Enablers

\section{Principle}

\section{Capability}

- Concern about speed of development, safety, and approval

- Perceptions about natural immunity
Principle 1: Clarify how vaccines were able to be developed and approved both quickly and safely
Tips for primary care/ communities:

- Explain speed of vaccine development: Explain that typical vaccine development bottlenecks include lack of funding and difficulty recruiting participants to clinical trials. COVID-19 vaccines build on years of vaccine research (i.e., not from scratch), had lots of funding and the world's best scientists, governments, universities, and pharmaceutical companies who immediately mobilized their resources in pursuit of protecting the world from COVID-19. Clarify that because so many participants volunteered to be in clinical trials, these large trials ran more quickly than usual because they could rapidly recruit large samples worldwide. ${ }^{11}$ This is why we were able to to get the well-tested, safe and effective vaccines to the public quickly

- Explain speed of vaccine approval: Health Canada is independent, and its mandate includes ensuring the safety of vaccines for Canadians. COVID-19 vaccines received priority in the review process, whereas vaccines for less transmissible diseases are required to wait in a queue. Health Canada shortened the administrative and organizational processes, but the safety standards were just as strict as for any other vaccine ${ }^{11}$

- Robust international and national system for monitoring and updating guidance for Ontarians. 
- Counteract misinformation about speed of development by sharing key facts (i.e., "The vaccine technology is new but it is built upon years of research which enabled rapid development, safety review and approval"'). ${ }^{12}$ Avoid repeating the misinformation itself, which can make incorrect statements seem familiar and true

\section{Opportunity}

- Seeing others similar to oneself encourages motivation to act

- Health care professionals are primary trusted sources but not for all groups ${ }^{12}$; consider other trusted sources tailored to groups (e.g. faith leaders, community leaders, elders)
- Medical mistrust
Principle 2: Build social norms through social comparison to similar others and leverage trusted sources (note these are different individuals in different communities) ${ }^{5}$
Tips for public health/communities:

- Leverage narrative and contextual anecdotes tailored to particular contexts and experiences

- Show people that different people identify with getting vaccinated

- Public commitment to vaccination through social proof (e.g., update social media profiles with messages such as "When it's my turn, I'm getting vaccinated", "I am vaccinated against COVID-19") $)^{13}$

Tips for government:

- Amplify when vaccination is accelerating, in general and for specific groups, generally and in specific jurisdictions

Tips for primary care/government/ communities:

- A health care professiona recommendation can increase beliefs in the vaccine's safety and reduce concerns about harm ${ }^{14}$

- Provision of culturally appropriate, multilingual vaccine communication materials online and in commonly accessed physical locations (e.g. vaccine clinics) ${ }^{12}$

Principle 3: Recognise the role that medical mistrust plays and allow time to address it

- Racialized communities, Indigenous

\section{Tips for public/community leaders:} peoples, immigrants, LGBTQ2S+ individuals, precariously housed people, people with disabilities and other marginalized groups traditionally face obstacles and inequalities in healthcare and this has been exacerbated by this pandemic. ${ }^{15-17}$ They may also have collective histories of experience with medical malpractice that affect current trust. Tailoring messages to these marginalized groups requires community engagement and cultural understanding 


\section{Motivation}

- Side effects in general

- Side effects of particular vaccines

- Worry about long-term side effects

- Side effects of getting COVID-19

- Raising threat without providing resources to address it may lead to disengaging

- Lack of clarity and consistency of messaging on effectiveness of different vaccines and assumptions about 'better' vaccines, including in light of VOCs
- Promoting intrinsic motivation may not be enough for some; leveraging extrinsic motivators may help when paired with other strategies

- Travel, work, family, socializing
Principle 4: Use effective risk communication tools to clarify risk and severity of getting COVID-19 and any vaccine side effects ${ }^{18}$

Principle 5: Consider incentives and contingent rewards that link vaccination to personal values and priorities ${ }^{19,20}$
Tips for government/communities:

- Be transparent about what is currently known and what is still being evaluated - evidence on vaccine effectiveness against variants is evolving daily and we are working with the information at hand. Acknowledge the possibility of varying effects or possible side effects to avoid further loss of trust if or when unforeseen events happen ${ }^{16}$

- Indicate that short-term side effects such as fever and muscle aches are expected as a part of the body's natural immune response to vaccine (e.g., this is the body working to make the protection against the virus)

- Monitor data on vaccine safety and side effects and make this information publicly available on a regular basis ${ }^{16}$

Tips for primary care/government/ communities:

- Pair information re: risk of VITT with key symptoms, what to do, and the effectiveness of treatment for VITT. Do not focus on the worst outcome in the absence of education on what the public can do about it

- Acknowledge that short-term side effects are common and inconvenient, while long-term or serious effects are extremely rare

Tips for public health/community leaders:

- Do not rely only on providing quantitative risk information; consider health literacy and numeracy across the population and use effective alternative means of presenting risk information beyond proportions

- Ensure trusted and/or similar others from diverse communities communicate risk information, who will not be the same for everyone

Tips for organizations:

- At vaccination clinics, offer free food/ non-alcoholic beverages; prize draws; altruistic raffles (50/50 donations to important causes); vouchers for $\%$ off for stores and venues that people frequent and are looking to get back to (e.g., sports venues)

Tips for public/communities/ governments:

- Getting vaccinated can help society open as soon as possible, as seen in countries with faster vaccine roll-outs, such as Israel, the UK and US. As this happens, build social norms around the return to activity for those who are vaccinated 


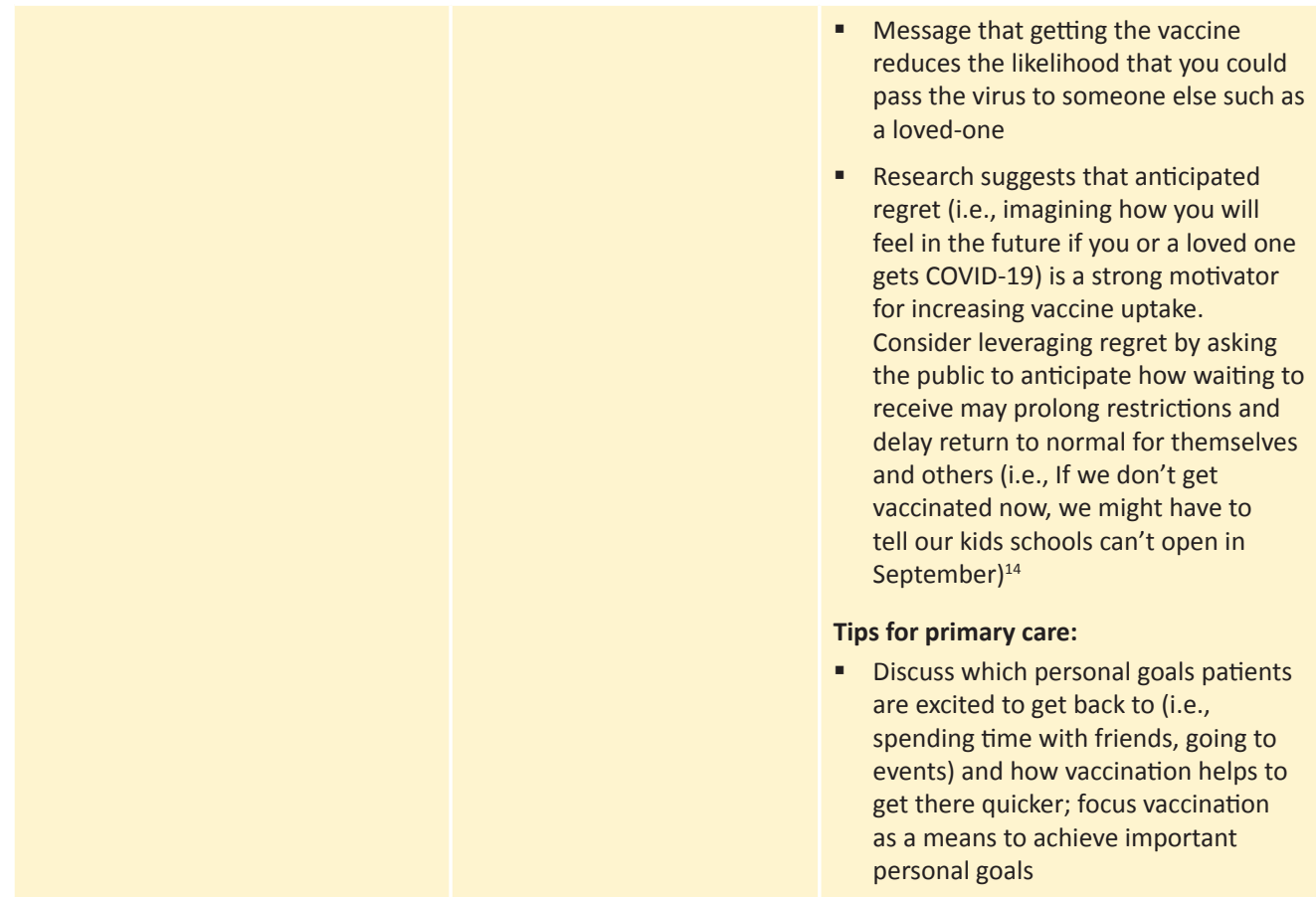

Table 2. Five Principles for Supporting the Decision to Get Vaccinated

\begin{tabular}{|c|c|c|}
\hline $\begin{array}{c}\text { Vaccination Uptake } \\
\text { Barriers/Enablers }\end{array}$ & Principle & Strategy in Action \\
\hline \multicolumn{3}{|c|}{ Capability } \\
\hline $\begin{array}{l}\text { - Forgetting to get vaccinated }{ }^{21} \\
\text { - Personal, social and societal } \\
\text { barriers }\end{array}$ & $\begin{array}{l}\text { Principle 1: Provide } \\
\text { reminders for each dose } \\
\text { and create a plan to address } \\
\text { anticipated obstacles to } \\
\text { booking and attending }\end{array}$ & $\begin{array}{l}\text { Tips for government/communities: } \\
\text { - Text messages, emails, phone calls from } \\
\text { trusted source prior to a vaccination } \\
\text { appointment with timing that balances } \\
\text { the potential need to mobilize support } \\
\text { in order to attend vs too early to be a } \\
\text { reminder }{ }^{22} \\
\text { - Reminding people over the phone may } \\
\text { be more effective than other types of } \\
\text { reminders (e.g., automatic calls, sending } \\
\text { a letter or postcard, sending a text } \\
\text { message) in increasing vaccine uptake } \\
\text { - Assume summer will be busier (e.g., } \\
\text { staycations) and accommodate option of } \\
\text { easily rescheduling } 2 \text { nd dose if needed }\end{array}$ \\
\hline \multicolumn{3}{|c|}{ Opportunity } \\
\hline $\begin{array}{l}\text { Majority of Ontarians want } \\
\text { to get vaccinated; assume } \\
\text { they want it unless otherwise } \\
\text { indicated }\end{array}$ & $\begin{array}{l}\text { Principle } 2 \text { : Nudge } \\
\text { vaccination by using opt out }\end{array}$ & $\begin{array}{l}\text { Tips for government: } \\
\text { - Messaging reminders in terms of "your } \\
\text { COVID-19 vaccination spot has been } \\
\text { reserved for you"22 }\end{array}$ \\
\hline
\end{tabular}


- Standard business hours make it difficult or impossible to attend due to work or family commitments

- Booking and bringing people safely to get vaccinated who might not otherwise be able to

- Booking systems difficult to use and navigate

- Location of vaccination clinic difficult to get to
Principle 3: Make booking and attendance easy by maximizing use of accessible and convenient locations, times and days of the week. Implement flexible work hours and paid time off to encourage uptake ${ }^{24,25}$

Tips for communities/government:

- Consider extended hours and nontraditional hours (including overnight or weekends) e.g. "Doses after dark" marathon vaccination clinic in Peel (for 32-hour non-stop) $)^{10}$

- Booking and attendance

- Childcare or ability to safely bring children (dedicated child friendly timing)

- Support for less tech savvy

- Offer transportation services to clinics for people unable to access or afford existing methods of transportation (i.e., older residents, those with disabilities, low-income individuals) ${ }^{17,26}$

\section{Tips for government:}

- Consent and online system/ allow everyone to pre-register for the vaccine

- Flag grassroots groups like VaccineHunters; https://vaccine-gta.ca/; indicates opportunity for government/ PHUs partnership

- Pop-ups, local plans, onsite vaccination at workplaces and apartment complexes $^{27}$

- Apply successes in other jurisdictions, including multiple decentralized vaccination sites to enhance access and communication strategies tailored to specific subgroups to increase vaccine confidence ${ }^{7}$

- Pop-ups at trusted community locations

- Clinics in familiar and accessible locations (e.g., pharmacies) may increase uptake in those that are hesitant ${ }^{23}$

\section{Tips for employers:}

- Partner with hospitals/public health units to host vaccination clinics that are convenient to employees

Tips for government/employers:

- Enable paid time off to get vaccinated or other means to support employees to reduce barriers to getting vaccinated

- Mandate employers to provide paid time off for vaccination

- Financially support transportation to off-site vaccination clinics

- Provide flexible leave policies for those who may have post-vaccination symptoms

Principle 4: One size will not fit all. Ensure multiple strategies to reach those that are homebound or not comfortable with technology

\section{Tips for government/communities:}

- Data from Ontario suggest that vaccine intentions vary by age, ethnicity, region and profession. Authentically work with these communities to address barriers to vaccination (Murty K, personal communication)

- Bring to isolated older adults in buildings or still living at home ${ }^{28}$

- Home visits for those not able to go to a vaccine clinic $^{29}$ 


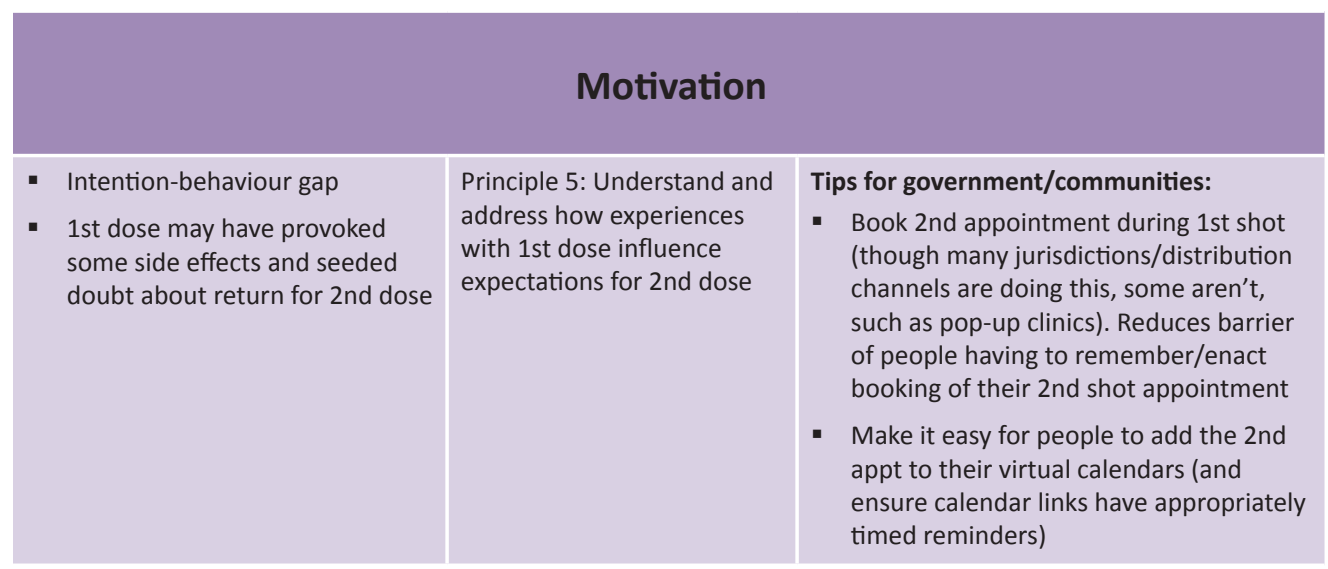

Table 3. Five Principles for Supporting Vaccination for Each Dose

\section{Interpretation}

Behavioural science helps us understand that Ontario's path to COVID-19 immunity involves multiple behavioural factors that can be effectively addressed if considered proactively. For some, logistics of the second dose have already been determined through pre-scheduling. For many, where and how they will receive the second dose remains unclear or challenged by the need for rebooking. For those who received dose one of the AstraZeneca vaccine, which vaccine they will receive for their second dose will require tailored communication in and of itself and weighing identified rare but serious side effects against the personal risk of COVID-19. ${ }^{11,30}$

Early efforts to distribute vaccine supply to increase opportunity highlight the value of such approaches and the importance of expanding the use of non-traditional distribution models moving forward to leverage community infrastructure and resources. These include strategies such as Peel Region's "Doses After Dark," outreach models to vaccinate homebound individuals, and arranging clinics in industrial areas or within high-occupancy workplaces. ${ }^{10,28}$

Evidence from other jurisdictions suggests that a substantial minority of those receiving the first dose are not returning for a second dose. ${ }^{31}$ Ontario should anticipate a similar pattern to mitigate its realization locally. Clarity on the need for and relative advantage of a second dose (i.e., long-term protection) as well as challenges to accessing the first dose (including logistical factors) may influence intention to get second dose. It is not a given that being offered the second dose - without any additional or different supports beyond those provided for the first dose - will lead Ontarians to followthrough with the full course of vaccination.

\section{Methods Used for This Science Brief}

To assess current vaccination intention, we drew upon existing Canadian and Ontario data as of April 28, 2021 from national and provincial surveys, including the Field's Institute Survey (Murty K, personal communication). To report on vaccination uptake, we used the Government of Canada vaccine coverage reports as of June 12, 2021. ${ }^{1}$

To address known barriers and enablers to vaccination intention, we used a behavioural science evidence synthesis by Crawshaw, Konnyu et al.'s (May 31, 2021) work collecting data on vaccination views post-vaccination approval, supplemented by Ontario-specific barrier/enablers from a representative provincial survey (Murty K, personal communication).

Finally, to identify strategies to enable and support vaccine uptake, we drew upon existing literature on strategies recommended and shown to be effective in increasing vaccination intention and uptake. This has included evidence syntheses produced by 
the Evidence Synthesis Network and Public Health Ontario, the WHO, and individual syntheses. ${ }^{14,32}$ We then used the Behaviour Change Wheel to map the identified barriers/ enablers, to nine specific categories of intervention functions (i.e., change strategies) and seven policy categories (i.e., policy levers). This analysis has allowed the Working Group to suggest fit-for-purpose behavioural solutions supported by policy to address specific particular barriers and enablers to vaccination uptake in Ontarians. ${ }^{2}$

\section{Author Contributions}

JP and LD conceived the Science Brief. JP, LD and AW wrote the first draft of the Science Brief. AW performed the analyses. All authors revised the Science Brief critically for important intellectual content and approved the final version.

JP acknowledges that part of the research informing this brief is informed by a living behavioural science evidence synthesis which is in part supported by a sub-grant from the Public Health Agency of Canada to COVID-END.

\section{References}

1. Canada PHA of. Demographics: COVID-19 vaccination coverage in Canada Canada.ca. aem. Published January 15, 2021. Accessed May 17, 2021. https:// health-infobase.canada.ca/covid-19/vaccination-coverage/

2. Michie S, van Stralen MM, West R. The behaviour change wheel: A new method for characterising and designing behaviour change interventions. Implement Sci. 2011;6(1):42. https://doi.org/10.1186/1748-5908-6-42

3. Chagla Z, Ma H, Sander B, Baral SD, Mishra S. Characterizing the disproportionate burden of SARS-CoV-2 variants of concern among essential workers in the Greater Toronto Area, Canada. medRxiv. Published online March 26, 2021:2021.03.22.21254127. https://doi.org/10.1101/2021.03.22.21254127

4. Sundaram ME, Calzavara A, Mishra $S$, et al. The individual and social determinants of COVID-19 in Ontario, Canada: a population-wide study. medRxiv. Published online November 12, 2020:2020.11.09.20223792. https://doi. org/10.1101/2020.11.09.20223792

5. Crawshaw J, Konnyu K, Castillo G, van Allen Z, Grimshaw JM, Presseau J. Factors affecting COVID-19 vaccination acceptance and uptake among the general public: a living behavioural science evidence synthesis (v2, May 31st, 2021). :76. https:// www.mcmasterforum.org/docs/default-source/product-documents/livingevidence-syntheses/covid-19-living-evidence-synthesis-4.2---factors-affectingcovid-19-vaccination-acceptance-and-uptake-among-the-general-public. pdf?sfvrsn=c3c4cb8a_5

6. Griffith J, Marani H, Monkman H. COVID-19 Vaccine Hesitancy in Canada: Content Analysis of Tweets Using the Theoretical Domains Framework. J Med Internet Res. 2021;23(4):e26874. https://doi.org/10.2196/26874

7. Choi Y, Stall NM, Maltsev A, et al. Lessons learned from Israel's vaccine rollout. Sci Briefs Ont COVID-19 Sci Advis Table. 2021;2(9). https://doi.org/10.47326/ ocsat.2021.02.09.1.0

8. Zuber MC. U.S. vaccine rollout vastly outpacing Canada's: what can we learn from American push? Coronavirus. Published February 21, 2021. Accessed May 17, 2021. https://www.ctvnews.ca/health/coronavirus/u-s-vaccine-rollout-vastlyoutpacing-canada-s-what-can-we-learn-from-american-push-1.5317867

9. The Chaotic Race to Vaccinate Peel. The Local. Published May 3, 2021. Accessed May 17, 2021. https://thelocal.to/the-chaotic-race-to-vaccinate-peel/ 
10. 'Doses After Dark' 32-hour vaccination clinic coming to Peel Region - Region of Peel. Accessed May 17, 2021. https://peelregion.ca/news/archiveitem. asp?year $=2021 \&$ month $=4 \&$ day $=10 \&$ file $=2021410 . x \mathrm{ml}$

11. Presseau J, Desveaux L, Allen U, et al. Behavioural science principles for supporting COVID-19 vaccine confidence and uptake among Ontario health care workers. Sci Briefs Ont COVID-19 Sci Advis Table. 2021;2(12). https://doi.org/10.47326/ ocsat.2021.02.12.1.0

12. Ontario Agency for Health Protection and Promotion (Public Health Ontario). Building confidence in vaccines. Toronto, ON: Queen's Printer for Ontario; 2021.14.

13. Wood S, Schulman K. Beyond Politics - Promoting Covid-19 Vaccination in the United States. N Engl J Med. 2021;384(7):e23. https://doi.org/10.1056/ NEJMms2033790

14. Brewer NT, Chapman GB, Rothman AJ, Leask J, Kempe A. Increasing Vaccination: Putting Psychological Science Into Action. Psychol Sci Public Interest J Am Psychol Soc. 2017;18(3):149-207. https://doi.org/10.1177/1529100618760521

15. Brown KA, Stall NM, Joh E, et al. A strategy for the mass distribution of COVID-19 vaccines in Ontario based on age and neighbourhood. Sci Briefs Ont COVID-19 Sci Advis Table. 2021;2(10). https://doi.org/10.47326/ocsat.2021.02.10.1.0

16. Konnyu KJ, Benitez G. What are the barriers and facilitators to individuals' willingness to be vaccinated for COVID-19? I Center for Evidence Synthesis in Health | Brown University. brown.edu. Published January 20, 2021. https:// www.brown.edu/public-health/cesh/news/2021/01/what-are-barriers-andfacilitators-individuals\%E2\%80\%99-willingness-be-vaccinated-covid-19

17. Rotenberg S, Downer MB, Brown H, et al. COVID-19 vaccination for people with disabilities. Sci Briefs Ont COVID-19 Sci Advis Table. 2021;2(35). https://doi. org/10.47326/ocsat.2021.02.35.1.0

18. Canada PHA of. COVID-19 vaccine safety: Weekly report on side effects following immunization. aem. Published January 8, 2021. Accessed May 17, 2021. https:// health-infobase.canada.ca/covid-19/vaccine-safety/\#a3

19. Mantzari E, Vogt F, Marteau TM. Financial Incentives for Increasing Uptake of HPV Vaccinations: A Randomized Controlled Trial. Health Psychol. 2015;34(2):160-171. https://doi.org/10.1037/hea0000088

20. Wigham S, Ternent L, Bryant A, Robalino S, Sniehotta FF, Adams J. Parental financial incentives for increasing preschool vaccination uptake: systematic review. Pediatrics. 2014;134(4):e1117-1128. https://doi.org/10.1542/peds.2014-1279

21. Vann JCJ, Jacobson RM, Coyne-Beasley T, Asafu-Adjei JK, Szilagyi PG. Patient reminder and recall interventions to improve immunization rates. Cochrane Database Syst Rev. 2018;(1). https://doi.org/10.1002/14651858.CD003941.pub3

22. Milkman KL, Patel MS, Gandhi L, et al. A megastudy of text-based nudges encouraging patients to get vaccinated at an upcoming doctor's appointment. Proc Natl Acad Sci U S A. 2021;118(20). https://doi.org/10.1073/pnas.2101165118

23. Strategies to Encourage Vaccine Acceptance and Address Vaccine Hesitancy Evidence Synthesis Network. Accessed May 17, 2021. https://esnetwork.ca/ briefings/strategies-to-encourage-vaccine-acceptance-and-address-vaccinehesitancy/

24. COVID-19 vaccine refusal, UK - Office for National Statistics. Accessed May 17, 2021. https://www.ons.gov.uk/peoplepopulationandcommunity/healthandsocialcare/ 
healthandwellbeing/bulletins/covid19vaccinerefusaluk/februarytomarch2021

25. Thompson A, Stall NM, Born KB, et al. Benefits of Paid Sick Leave During the COVID-19 Pandemic. Ontario COVID-19 Science Advisory Table; 2021. https://doi. org/10.47326/ocsat.2021.02.25.1.0

26. COVID-19: How to Get Vaccinated - City of Toronto. Accessed May 17, 2021. https://www.toronto.ca/home/covid-19/covid-19-protect-yourself-others/covid19-vaccines/covid-19-how-to-get-vaccinated/?accordion=city-immunizationclinics

27. Mishra S, Stall NM, Ma H, et al. A vaccination strategy for Ontario COVID-19 hotspots and essential workers. Sci Briefs Ont COVID-19 Sci Advis Table. 2021;2(26). https://doi.org/10.47326/ocsat.2021.02.26.1.0

28. Huynh T, Sava N, Hahn-Goldberg S, et al. Mobile on-site COVID-19 vaccination of naturally occurring retirement communities by neighbourhood risk in Toronto. Sci Briefs Ont COVID-19 Sci Advis Table. 2021;1(14). https://doi.org/10.47326/ ocsat.2021.02.14.1.0

29. Stall NM, Nakamachi Y, Chang M, et al. Mobile in-home COVID-19 vaccination of Ontario homebound older adults by neighbourhood risk. Sci Briefs Ont COVID-19 Sci Advis Table. 2021;1(19). https://doi.org/10.47326/ocsat.2021.02.19.1.0

30. Pai M, Stall NM, Grill A, et al. Vaccine-Induced Immune Thrombotic Thrombocytopenia (VITT) following adenovirus vector COVID-19 vaccination. Sci Briefs Ont COVID-19 Sci Advis Table. 2021;2(17). https://doi.org/10.47326/ ocsat.2021.02.17.2.0

31. Waldrop T. Why so many Americans are skipping their second COVID-19 shot. Coronavirus. Published May 2, 2021. Accessed May 17, 2021. https://www. ctvnews.ca/health/coronavirus/why-so-many-americans-are-skipping-theirsecond-covid-19-shot-1.5410984

32. Lawes-Wickwar S, Ghio D, Tang MY, et al. A Rapid Systematic Review of Public Responses to Health Messages Encouraging Vaccination against Infectious Diseases in a Pandemic or Epidemic. Vaccines. 2021;9(2). https://doi.org/10.3390/ vaccines 9020072 